\title{
Arzneimittelsicherheit im Alter - wissen wir genug über die Arzneimitteltherapie bei älteren Patienten?
}

Arzneimitteltherapie bei älteren Patienten ist die Regel, nicht die Ausnahme: Die überwiegende Zahl an Medikamenten wird von Menschen eingenommen, die über 65 Jahre alt sind. In Untersuchungen in Krankenhausnotaufnahmen zum Aufnahmegrund war das durchschnittliche Alter von Patienten, die aufgrund einer Arzneimittelnebenwirkung die Notaufnahme aufgesucht hatten, 75 Jahre, und es wurden im Mittel 7 Arzneimittel gleichzeitig täglich eingenommen.

Wenn ältere Patienten auch die Hauptkundengruppe in den Apotheken darstellen, ist die Frage, ob wir genug über die Sicherheit der Arzneimitteltherapie bei ihnen wissen, durchaus berechtigt.

In der Zulassung und klinischen Arzneimittelentwicklung werden nach wie vor hauptsächlich Patienten unter 65 Jahren untersucht, und der höhere Grad an Offlabel-Gebrauch bei älteren Patienten, wie auch die vermehrt bei Polypharmazie auftretenden Arzneimittelwechselwirkungen, tragen dazu bei, dass im Alter erhöhte Nebenwirkungsrisiken bestehen.

Zwar wissen wir einiges über die geänderte Pharmakologie im Alter, jedoch sind wir noch nicht in der Lage, die Sicherheit von Arzneimitteltherapien genau einzuschätzen oder individuell vorherzusagen, was die hohe Anzahl an unerwünschten Arzneimittelwirkungen (UAW) von 6,5\% der Krankenhausnotaufnahmen verdeutlichen mag. Die Ursachen für UAW im Alter sind vielfältig und zum einen durch das besondere Vulnerabilitätsprofil bedingt, zum anderen durch die pharmakokinetischen Eigenschaften und Wechselwirkungen zwischen den Substanzen, die im Alter, bedingt durch die veränderte Physiologie des Menschen unterschiedlich ausfallen. AnaIysen von UAW aus unseren Studien illustrieren dies.

Gerade im Zeitalter der digitalen Daten und Dokumentationsmöglichkeiten sollte die Datengenerierung aus klinischer Forschung und Versorgung eine breitere Evi- denzbasis schaffen, sodass wir in Zukunft genug über Arzneimittelsicherheit im Alter wissen, um die Arzneimitteltherapie bei älteren Patienten individuell gestalten zu können.

\section{Interessenkonflikt}

Die Autorin gibt an, dass kein Interessenkonflikt besteht.

\section{Autorinnen/Autoren}

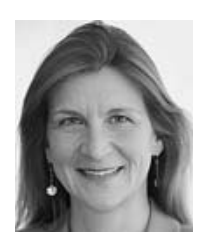

\section{Julia Stingl}

Univ. Prof. Dr. med., Institut für Klinische Pharmakologie, Universitätsklinikum der RWTH Aachen (UKA)

\section{Korrespondenzadresse}

Univ. Prof. Dr. med. Julia Stingl Institut für Klinische Pharmakologie Universitätsklinikum der RWTH Aachen (UKA)

Wendlingweg 2

52074 Aachen

Jstingl@ukaachen.de 